Review

\title{
Continuous-Variable Entanglement Swapping
}

Kevin Marshall ${ }^{1, *}$ and Christian Weedbrook ${ }^{2}$

${ }^{1}$ Department of Physics, University of Toronto, Toronto, M5S 1A7, Canada

${ }^{2}$ QKD Corp., 60 St. George St., Toronto, M5S 1A7, Canada; E-Mail: christian.weedbrook@gmail.com

* Author to whom correspondence should be addressed; E-Mail: marshall@physics.utoronto.ca.

Received: 26 March 2015 / Accepted: 4 May 2015 / Published: 13 May 2015

\begin{abstract}
We present a very brief overview of entanglement swapping as it relates to continuous-variable quantum information. The technical background required is discussed and the natural link to quantum teleportation is established before discussing the nature of Gaussian entanglement swapping. The limitations of Gaussian swapping are introduced, along with the general applications of swapping in the context of to quantum communication and entanglement distribution. In light of this, we briefly summarize a collection of entanglement swapping schemes which incorporate a non-Gaussian ingredient and the benefits of such schemes are noted. Finally, we motivate the need to further study and develop such schemes by highlighting requirements of a continuous-variable repeater.
\end{abstract}

Keywords: continuous variables; entanglement swapping; quantum teleportation

\section{Introduction}

Information permeates through most, if not all, aspects of our everyday life. Sometimes it's easy to forget the value our culture places on information; for example, a fraction of a second can make the difference between a profit or a loss as it does in high-frequency trading [1]. Communicating privately in the presence of an adversary makes possible the on-line transactions we take for granted; however, the methods we currently use to accomplish this rely on an assumption of the computational difficulty of some problem. Quantum key distribution (QKD) harnesses the inherently "weird” properties of quantum theory to provide a method of privately communicating which is based not on some assumption, but instead on the laws of physics as we understand them [2,3]. 
An important primitive in quantum communication is that of quantum teleportation $[4,5]$; this provides a method of transferring an unknown quantum state from one party, Alice, to another, Bob, given that they already share an entangled state. A closely related primitive is that of entanglement swapping [6-8]. Suppose Alice and Bob share an entangled pair and Bob uses quantum teleportation to teleport his half to a third party, Charlie. It can be shown that the resulting state between Alice and Charlie is entangled. The ability to transfer the non-local correlations characterized by entanglement in such a fashion has applications in quantum communication [9-11], where one cannot simply amplify a quantum signal as one would do with a classical one.

In this paper we review entanglement swapping in the framework of continuous-variable (CV) quantum information and how it relates to recent advances in the field. The paper is structured as follows: In Section 2 we review the necessary formalism to discuss entanglement swapping. Next, we present the general CV swapping protocol in Section 3. We discuss the special case of Gaussian entanglement swapping in Section 3.1 in the covariance matrix framework. In Section 3.2 we discuss both hybrid and non-Gaussian protocols and summarize their differences from the Gaussian protocol. Finally, in Section 4, we summarize the various approaches and how such protocols are related to applications in the field.

\section{Formalism Review}

In this section, we develop the necessary formalism to discuss CV entanglement swapping. A CV system is a canonical infinite dimensional quantum system comprised of $N$ bosonic modes with an associated Hilbert space $\mathcal{H}=\otimes_{k=1}^{N} \mathcal{H}_{k}$. Each of these modes $\mathcal{H}_{k}$ has an associated annihilation and creation operator $\hat{a}, \hat{a}^{\dagger}$ respectively. These operators obey the commutation relations $\left[\hat{a}_{i}, \hat{a}_{j}^{\dagger}\right]=\delta_{i j}$ and $\left[\hat{a}_{i}, \hat{a}_{j}\right]=\left[\hat{a}_{i}^{\dagger}, \hat{a}_{j}^{\dagger}\right]=0$. The space $\mathcal{H}_{k}$ is spanned by the Fock basis $\left\{\left|n_{k}\right\rangle\right\}_{n=0}^{\infty}$ of eigenstates of the number operator $\hat{n}_{k}=\hat{a}_{k}^{\dagger} \hat{a}_{k}$. These eigenstates have the property that $\hat{n}|n\rangle=n|n\rangle, \hat{a}|n\rangle=\sqrt{n}|n-1\rangle$, $\hat{a}^{\dagger}|n\rangle=\sqrt{n+1}|n+1\rangle$, as well as the fact that the vacuum state $|0\rangle$ is annihilated by $\hat{a}|0\rangle=\mathbf{0}$. In the absence of any interactions, these modes evolve according to the Hamiltonian $H=\sum_{k=1}^{N}\left(\hat{a}_{k}^{\dagger} \hat{a}_{k}+1 / 2\right)$. We can define two quadrature operators $\hat{q}_{k}=\hat{a}_{k}+\hat{a}_{k}^{\dagger}$ and $\hat{p}_{k}=i\left(\hat{a}_{k}^{\dagger}-\hat{a}_{k}\right)$ which act in a similar fashion to the position and momentum operators in the quantum harmonic oscillator. These commutation relations can be compactly written by defining

$$
\hat{\mathbf{x}}=\left(\hat{q}_{1}, \hat{p}_{1}, \ldots, \hat{q}_{N}, \hat{p}_{N}\right)
$$

so that we have $\left[\hat{x}_{i}, \hat{x}_{j}\right]=2 i \Omega_{i j}$ where

$$
\Omega=\bigoplus_{k=1}^{N}\left(\begin{array}{cc}
0 & 1 \\
-1 & 0
\end{array}\right)
$$

is the symplectic form.

Of particular interest in CV systems are Gaussian states and operations [12]. A Gaussian state, with density matrix $\hat{\rho}$, is completely characterized by its first two moments; the displacement vector

$$
\overline{\mathbf{x}}=\langle\hat{\mathbf{x}}\rangle=\operatorname{Tr}(\hat{\mathbf{x}} \hat{\rho})
$$


and the covariance matrix

$$
V_{i j}=\frac{1}{2}\left\langle\Delta \hat{x}_{i}, \Delta \hat{x}_{j}\right\rangle
$$

where $\Delta \hat{x}_{i}=\hat{x}_{i}-\left\langle\hat{x}_{i}\right\rangle$ and $\{.,$.$\} is the anticommutator. In this form, the uncertainty relation can be$ expressed as $V+i \Omega \geq 0$. We can fully represent an arbitrary quantum state $\hat{\rho}$ by its Wigner function, defined as

$$
W(\mathbf{x})=\int_{\mathbb{R}^{2 N}} \frac{d^{2 N} \boldsymbol{\xi}}{(2 \pi)^{2 N}} \exp \left(-i \mathbf{x}^{T} \Omega \boldsymbol{\xi}\right) \chi(\boldsymbol{\xi}),
$$

where $\chi(\boldsymbol{\xi})=\operatorname{Tr}[\hat{\rho} D(\boldsymbol{\xi})]$ is the characteristic function and $D(\boldsymbol{\xi})=\exp \left(i \hat{\mathbf{x}}^{\mathbf{T}} \boldsymbol{\Omega} \boldsymbol{\xi}\right)$ is the Weyl operator. For Gaussian states the Wigner function can be written as

$$
W(\mathbf{x})=\frac{1}{(2 \pi)^{N} \sqrt{\operatorname{det} V}} e^{-(\mathbf{x}-\overline{\mathbf{x}}) V^{-1}(\mathbf{x}-\overline{\mathbf{x}}) / 2},
$$

and thus we have complete knowledge of the state from only the first two moments. It is convenient for many purposes to deal only with the displacement vector and covariance matrix directly with the knowledge that we can always express the state in another form if we so choose.

Gaussian operations are those that take Gaussian states to other Gaussian states. They correspond to Hamiltonians which are linear or quadratic in the quadrature operators. To every unitary transformation $U_{S, d}$ generated from a quadratic Hamiltonian there exists a symplectic operator $S$ and vector $\mathbf{d}$ which generate the mapping $\hat{\mathbf{x}} \rightarrow S \hat{\mathbf{x}}+\mathbf{d}$. This transforms the moments of a Gaussian state as $\overline{\mathbf{x}} \rightarrow S \overline{\mathbf{x}}+\mathbf{d}$ and $V \rightarrow S V S^{T}$.

The Einstein-Podolsky-Rosen (EPR) state

$$
|\lambda\rangle=\sqrt{1-\lambda^{2}} \sum_{n=0}^{\infty}(-\lambda)^{n}|n\rangle_{a}|n\rangle_{b},
$$

with $\lambda=\tanh r \in[0,1]$, where $r$ is the squeezing parameter, is one important example of a Gaussian state. This two-mode state is entangled, and often used as a resource state in CV protocols. The EPR state has zero mean and covariance matrix given by

$$
V_{E P R}=\left(\begin{array}{cc}
\nu \mathbb{I} & \sqrt{\nu^{2}-1} Z \\
\sqrt{\nu^{2}-1} Z & \nu \mathbb{I}
\end{array}\right),
$$

where $\nu=\cosh 2 r, \mathbb{I}$ is the $2 \times 2$ identity matrix and $Z$ is the Pauli matrix $Z=\operatorname{diag}(1,-1)$.

Quantum teleportation can be conveniently described in the Wigner function formalism, where one makes a Bell measurement between the input state and half of the entangled pair [5]. Suppose we have an input state described by a Wigner function $W_{i n}(\hat{x}, \hat{p})$ and an EPR pair described by $W_{E P R}\left(\hat{x}_{1}, \hat{p}_{1}, \hat{x}_{2}, \hat{p}_{2}\right)$. To teleport the state Alice mixes the input state and the first mode of the EPR pair on a 50:50 beam splitter which performs the transformations: $\hat{x} \rightarrow \hat{X}=\left(\hat{x}-\hat{x}_{1}\right) / \sqrt{2}, \hat{p} \rightarrow \hat{k}=\left(\hat{p}-\hat{p}_{1}\right) / \sqrt{2}, \hat{x}_{1} \rightarrow q=$ $\left(\hat{x}+\hat{x}_{1}\right) / \sqrt{2}$, and $\hat{p}_{1} \rightarrow \hat{P}=\left(\hat{p}+\hat{p}_{1}\right) / \sqrt{2}$. Alice then measures the pair $(\hat{X}, \hat{P})$ and sends the result $(\bar{X}, \bar{P})$ to Bob who displace his mode as $\hat{x}_{2} \rightarrow \hat{x}_{\text {out }}=\hat{x}_{2}+\sqrt{2} g \bar{X}$, for some possible gain $g>0$, and similarly for $\hat{p}_{\text {out }}$. The final state is obtained by integrating over all measurement outcomes as

$$
W_{\text {out }}(x, p)=\int d \bar{X} d k d q d \bar{P} W_{t}(\bar{X}, k, q, \bar{P}, x-\sqrt{2} g \bar{X}, p-\sqrt{2} g \bar{P}),
$$


where $W_{t}\left(\zeta, \zeta_{A}, \zeta_{B}\right)=W_{i n}\left[\left(\zeta+\zeta_{A}\right) / \sqrt{2}\right] W_{E P R}\left[\left(\zeta_{A}-\zeta\right) / \sqrt{2}, \zeta_{B}\right]$ is the Wigner function for the total state with $\zeta_{i}=\left(\hat{x}_{i}, \hat{p}_{i}\right)$.

\section{Entanglement Swapping}

The entanglement swapping procedure follows the spirit of teleportation as presented in the previous section, where one simply uses another entangled state as the input state; such schemes have been experimentally demonstrated [13,14]. One can allow for a more general protocol by including displacements on both remaining modes with different gains. Gain-tuning the displacements to improve the fidelity of teleportation has been previously studied in the literature [7,15]. Furthermore, one need not use an EPR state as the source of entanglement; one can consider protocols which use non-Gaussian entanglement or hybrid approaches which make use of both discrete and continuous elements [16].

\subsection{Gaussian}

In the case of Gaussian quantum information it is convenient to discuss entanglement swapping in the covariance matrix framework $[8,17]$. Supposed we have an entangled state with covariance matrix described by

$$
\sigma=\left(\begin{array}{cccc}
a & 0 & c_{+} & 0 \\
0 & a & 0 & c_{-} \\
c_{+} & 0 & b & 0 \\
0 & c_{-} & 0 & b
\end{array}\right)
$$

this is a suitable form to study as all two-mode Gaussian states can be brought into this form, with zero displacement vector, by local Gaussian operations [18]. If we consider swapping modes 2,3 for entangled pairs characterized by covariance matrices $\sigma_{12}$ and $\sigma_{34}$ we find that by choosing the gains on both remaining modes in an optimal fashion we obtain the new covariance matrix [19]

$$
\sigma_{o p t}=\left(\begin{array}{cccc}
a-\xi & 0 & \xi & 0 \\
0 & a-\xi & 0 & -\xi \\
\xi & 0 & b-\xi & 0 \\
0 & -\xi & 0 & b-\xi
\end{array}\right)
$$

where $\xi=c^{2} /(a+b)$ and where we have chosen $c_{+}=-c_{-}=c$ for simplicity.

Interestingly, it can be shown that the purity of the resulting state is equal to the purity of the initial state. This could be used, for example to obtain a state of higher purity over a lossy channel by sending half of two EPR pairs to the middle and swapping as opposed to directly sending half of an EPR pair over the full length of the channel. One would hope that this would be useful for building a CV repeater, however it has been shown that such direct transmission is always preferable for Gaussian entanglement distribution when compared to the swapping scheme considered above [19]. This is different from the case of discrete variable (DV) repeaters where swapping can actually be beneficial [20], therefore the first building block of a $\mathrm{CV}$ repeater must be a distillation step. 


\subsection{Non-Gaussian}

We present here a brief summary of some alternative entanglement swapping protocols which make use of non-Gaussian elements. Entanglement swapping is primarily useful as a primitive in quantum communication, however, as seen in the previous section, it is necessary for many protocols of interest to have a distillation process. An important result is that one cannot distill entanglement from Gaussian states with only Gaussian operations and measurements [21,22]. It is fruitful to then consider schemes which add a non-Gaussian element at some stage.

One such hybrid scheme was proposed by Loock et al. in 2006 [23]. In this scheme one mediates entanglement between atomic qubits with a dispersive light-matter interaction and by sending the resulting bright coherent states between stations. One advantage of this scheme is that the measured light observable is a continuous phase, as opposed to a discrete occupation, which places less stringent interferometric requirements on the experiment. Instead of performing the usual homodyne detections in the swapping protocol, it can be advantageous to consider performing an unambiguous state discrimination measurement. This measurement can allow one to eliminate bit-flip errors, however it requires photon detectors [24]. Ideally, such a method could allow for long-distance qubit communication with high fidelity.

Another scheme which uses CV states to mediate DV entanglement was considered by Takeda et al. [25]; this method has also been investigated experimentally [26]. This scheme is all optical and mediates photonic qubit entanglement, either single or dual-rail, through the use of CV entanglement in the form of EPR pairs. By judiciously choosing the gain, one is able to teleport DV entanglement for any non-zero amount of squeezing provided the loss on the initial states satisfies a constraint.

The reverse of this protocol has been studied by Andersen et al. where an all optical proposal for teleporting CV entanglement with DV resources is discussed [27]. To approach unit fidelity with Gaussian entanglement swapping one must continue to increase the squeezing of the EPR pairs, this limit is unphysical in the sense that perfect entanglement requires infinite energy. This scheme attempts to circumvent this issue by using a finite number of DV entangled pairs to faithfully teleport CV states.

A proposal by Brask et al. [28] investigates the nature of a CV repeater which uses non-Gaussian states but only Gaussian measurements. In this approach, one uses entangled coherent states superpositions, known as "Schrödinger cat states" to distribute entanglement. These states are useful for a variety of quantum information protocols including fault-tolerant quantum computation [29] and teleportation [30-32]. This scheme takes advantage of the deterministic nature of CVs and readily available detectors to attempt to provide an experimentally feasible approach which is capable of achieving similar rates to atomic-ensemble repeaters at long distances. The entanglement swapping relies on the familiar Bell state measurement with homodyne detectors, and can be made near deterministic with additional single-mode resource cat states.

It is also possible to simply take the Gaussian entanglement swapping protocol and add a non-Gaussian element, such as photon subtraction or replacement as a distillation step. Such a notion has been considered in detail as it relates to the notion of a CV repeater [33]. It is shown that one can extend the maximum distance over which one can extract a secret-key from a noisy channel by using such a repeater architecture. 


\section{Summary}

We have presented a collection, but by no means all, of CV entanglement swapping protocols and briefly discussed their application to quantum key distribution and the notion of distillation and quantum repeaters. While Gaussian entanglement swapping is desirable for its simplicity, one requires non-Gaussian elements if one hopes to distill entanglement. Thus we presented a host of protocols which make use of non-Gaussian states, either discrete or continuous variables, and which offer a range of benefits. Such protocols are important in the advancement of continuous-variable quantum communication, where there has been limited work on continuous-variable repeaters such as a full rate analysis. We know that in order to achieve high key-rates over long distances we need repeaters; there is a fundamental limit to the achievable rate over a lossy channel, i.e., its private capacity, which lies between the lower bound of Ref. [34] and the upper bound of Ref. [35]. Furthermore, we know such repeater stations must include a non-Gaussian element [36]. It will therefore be potentially fruitful going forward to build off of the host of entanglement swapping protocols that exist in the literature today.

\section{Conflicts of Interest}

The authors declare no conflict of interest.

\section{References}

1. Lewis, M. Flash Boys: A Wall Street Revolt; WW Norton \& Company: New York, NY, USA, 2014.

2. Bennett, C.H.; Brassard, G. Quantum cryptography: Public key distribution and coin tossing. In Proceedings of IEEE International Conference on Computers, Systems, and Signal Processing, Bangalore, India, 10-19 December 1984; p. 175.

3. Grosshans, F.; Grangier, P. Continuous Variable Quantum Cryptography Using Coherent States. Phys. Rev. Lett. 2002, 88, 057902.

4. Bennett, C.H.; Brassard, G.; Crépeau, C.; Jozsa, R.; Peres, A.; Wootters, W.K. Teleporting an unknown quantum state via dual classical and Einstein-Podolsky-Rosen channels. Phys. Rev. Lett. 1993, 70, 1895-1899.

5. Braunstein, S.L.; Kimble, H.J. Teleportation of Continuous Quantum Variables. Phys. Rev. Lett. 1998, 80, 869-872.

6. Żukowski, M.; Zeilinger, A.; Horne, M.A.; Ekert, A.K. "Event-ready-detectors" Bell experiment via entanglement swapping. Phys. Rev. Lett. 1993, 71, 4287-4290.

7. Polkinghorne, R.E.S.; Ralph, T.C. Continuous Variable Entanglement Swapping. Phys. Rev. Lett. 1999, 83, 2095-2099.

8. Pirandola, S.; Vitali, D.; Tombesi, P.; Lloyd, S. Macroscopic Entanglement by Entanglement Swapping. Phys. Rev. Lett. 2006, 97, 150403.

9. Briegel, H.J.; Dür, W.; Cirac, J.I.; Zoller, P. Quantum Repeaters: The Role of Imperfect Local Operations in Quantum Communication. Phys. Rev. Lett. 1998, 81, 5932-5935.

10. Braunstein, S.L.; Pirandola, S. Side-Channel-Free Quantum Key Distribution. Phys. Rev. Lett. 2012, 108, 130502. 
11. Lo, H.K.; Curty, M.; Qi, B. Measurement-Device-Independent Quantum Key Distribution. Phys. Rev. Lett. 2012, 108, 130503.

12. Weedbrook, C.; Pirandola, S.; García-Patrón, R.; Cerf, N.J.; Ralph, T.C.; Shapiro, J.H.; Lloyd, S. Gaussian quantum information. Rev. Mod. Phys. 2012, 84, 621-669.

13. Jia, X.; Su, X.; Pan, Q.; Gao, J.; Xie, C.; Peng, K. Experimental Demonstration of Unconditional Entanglement Swapping for Continuous Variables. Phys. Rev. Lett. 2004, 93, 250503.

14. Takei, N.; Yonezawa, H.; Aoki, T.; Furusawa, A. High-Fidelity Teleportation beyond the No-Cloning Limit and Entanglement Swapping for Continuous Variables. Phys. Rev. Lett. 2005, 94, 220502.

15. Ide, T.; Hofmann, H.F.; Furusawa, A.; Kobayashi, T. Gain tuning and fidelity in continuous-variable quantum teleportation. Phys. Rev. A 2002, 65, 062303.

16. Furusawa, A.; van Loock, P. Quantum Teleportation and Entanglement: A hybrid Approach to Optical Quantum Information Processing; Wiley: Weinheim, Germany, 2011.

17. Abdi, M.; Pirandola, S.; Tombesi, P.; Vitali, D. Continuous-variable-entanglement swapping and its local certification: Entangling distant mechanical modes. Phys. Rev. A 2014, 89, 022331.

18. Duan, L.M.; Giedke, G.; Cirac, J.I.; Zoller, P. Inseparability Criterion for Continuous Variable Systems. Phys. Rev. Lett. 2000, 84, 2722-2725.

19. Hoelscher-Obermaier, J.; van Loock, P. Optimal Gaussian entanglement swapping. Phys. Rev. A 2011, 83, 012319.

20. Collins, D.; Gisin, N.; De Riedmatten, H. Quantum relays for long distance quantum cryptography. J Mod. Opt. 2005, 52, 735-753.

21. Eisert, J.; Scheel, S.; Plenio, M.B. Distilling Gaussian States with Gaussian Operations is Impossible. Phys. Rev. Lett. 2002, 89, 137903.

22. Fiurášek, J. Gaussian Transformations and Distillation of Entangled Gaussian States. Phys. Rev. Lett. 2002, 89, 137904.

23. Van Loock, P.; Ladd, T.D.; Sanaka, K.; Yamaguchi, F.; Nemoto, K.; Munro, W.J.; Yamamoto, Y. Hybrid Quantum Repeater Using Bright Coherent Light. Phys. Rev. Lett. 2006, 96, 240501.

24. Van Loock, P.; Lütkenhaus, N.; Munro, W.J.; Nemoto, K. Quantum repeaters using coherent-state communication. Phys. Rev. A 2008, 78, 062319.

25. Takeda, S.; Mizuta, T.; Fuwa, M.; Yonezawa, H.; van Loock, P.; Furusawa, A. Gain tuning for continuous-variable quantum teleportation of discrete-variable states. Phys. Rev. A 2013, 88, 042327.

26. Takeda, S.; Mizuta, T.; Fuwa, M.; van Loock, P.; Furusawa, A. Deterministic quantum teleportation of photonic quantum bits by a hybrid technique. Nature 2013, 500, 315-318.

27. Andersen, U.L.; Ralph, T.C. High-Fidelity Teleportation of Continuous-Variable Quantum States Using Delocalized Single Photons. Phys. Rev. Lett. 2013, 111, 050504.

28. Brask, J.B.; Rigas, I.; Polzik, E.S.; Andersen, U.L.; Sørensen, A.S. Hybrid Long-Distance Entanglement Distribution Protocol. Phys. Rev. Lett. 2010, 105, 160501.

29. Lund, A.P.; Ralph, T.C.; Haselgrove, H.L. Fault-Tolerant Linear Optical Quantum Computing with Small-Amplitude Coherent States. Phys. Rev. Lett. 2008, 100, 030503. 
30. Van Enk, S.J.; Hirota, O. Entangled coherent states: Teleportation and decoherence. Phys. Rev. A 2001, 64, 022313.

31. Jeong, H.; Kim, M.S.; Lee, J. Quantum-information processing for a coherent superposition state via a mixedentangled coherent channel. Phys. Rev. A 2001, 64, 052308.

32. An, N.B.; Phien, H.N. Teleportation of a cat-state via attenuated quantum channel using only threshold detectors. Physics Letters A 2008, 372, 5666-5670.

33. Campbell, E.T.; Genoni, M.G.; Eisert, J. Continuous-variable entanglement distillation and noncommutative central limit theorems. Phys. Rev. A 2013, 87, 042330.

34. Pirandola, S.; García-Patrón, R.; Braunstein, S.L.; Lloyd, S. Direct and Reverse Secret-Key Capacities of a Quantum Channel. Phys. Rev. Lett. 2009, 102, 050503.

35. Takeoka, M.; Guha, S.; Wilde, M.M. Fundamental rate-loss tradeoff for optical quantum key distribution. Nat. Commun. 2014, 5, doi:10.1038/ncomms6235.

36. Namiki, R.; Gittsovich, O.; Guha, S.; Lütkenhaus, N. Gaussian-only regenerative stations cannot act as quantum repeaters. Phys. Rev. A 2014, 90, 062316.

(c) 2015 by the authors; licensee MDPI, Basel, Switzerland. This article is an open access article distributed under the terms and conditions of the Creative Commons Attribution license (http://creativecommons.org/licenses/by/4.0/). 